Relations industrielles

Industrial Relations

\title{
Propos sur le travail humain
}

\section{Pie XII}

Volume 13, numéro 2, avril 1958

URI : https://id.erudit.org/iderudit/1022448ar

DOI : https://doi.org/10.7202/1022448ar

Aller au sommaire du numéro

Éditeur(s)

Département des relations industrielles de l’Université Laval

ISSN

0034-379X (imprimé)

1703-8138 (numérique)

Découvrir la revue

Citer ce document

XII, P. (1958). Propos sur le travail humain. Relations industrielles / Industrial Relations, 13(2), 232-234. https://doi.org/10.7202/1022448ar
Résumé de l'article

Sa Sainteté le Pape Pie XII a reçu en audience, 10 janvier 1958, les délégués de la Commission consultative internationale des entrepreneurs de l'industrie chimique d'une douzaine de pays. Le Saint-Père, au cours de l'audience, a prononcé l'allocution dont nous reproduisons le texte et qui traite des aspects sociaux de l'important secteur d'activité qu'exercent les dirigeants de l'industrie chimique. 


\title{
MURDOCHVILLE: DERNIÈRE PIÈCE AU DOSSIER
}

\begin{abstract}
Le 11 février 1958, la CRO rejetait la seconde requête en certification des Métallurgistes unis d'Amérique (CTC) de Murdochville (Gaspe Copper Mines), décidant que le syndicat avait manifesté sa mauvaise foi en déclenchant une grève illégale durant les procédures de certification, en enfreignant un arrêt judiciaire interdisant le piquetage (alors que les tribunaux ne s'étaient pas encore prononcés sur l'injonction obtenue par la Compagnie), et en empêchant des non-grévistes de pénétrer dans la mine. Cette seconde requête avait été présentée en décembre 1957. A la suite de la décision de la CRO, MM. Gérard Picard et Roger Provost, présidents respectivement de la CTCC et de la FTQ, ont fait les commentaires suivants :
\end{abstract}

La Commission de relations ouvrières de la province vient de donner le coup de grâce à un syndicat ouvrier et confirme, par le fait même, que la loi actuelle, les délais qu'elle provoque et les procédures dilatoires qu'elle facilite, paralysent l'exercice du droit d'association des travailleurs.

La Fédération des travailleurs du Québec et la Confédération des travailleurs catholiques du Canada se joignent au Congrès du travail du Canada et au Symdicat des métallurgistes unis d'Amérique pour protester énergiquement contre cet état de chose.

Le droit d'association est un droit naturel inaliénable lorsqu'on l'exerce en poursuivant des fins légitimes. Dans le cas qui nous occupe, on s'en souvient, les mineurs de la Gaspe Copper Mines à Murdochville avaient presque tous donnó leur adhésion au Syndicat des métallurgistes unis d'Amérique il y a près de deux ans. La Gaspe Copper Mines, par des procédures dilatoires, a utilisé la loi pour empêcher l'exercice d'un droit fondamental. Les mineurs ont ainsi été poussés à la grève en vue de saisir l'opinion publique du soandale de Murdochville. La tragique réalité fait ressortir que la Gaspe Copper Mines, le gouvernement provincial, la police provinciale, les briseurs de grève, et la Commission de relations ouvrières se sont ligués pour empêcher les mineurs de Murdochville d'être représentés par le syndicat de leur choix.

Une injustice aussi criante doit réveiller non seulement tous les travailleurs mais encore tous les autres citoyens qui restent sensibles à la défense des droits de l'homme. Nos institutions démocratiques ne devraient pas permettre que des tragédies comme Murdochville, où il $\mathrm{y}$ a eu des morts et des blessés, se répètent.

Le dossier est déjà chargé, dans la province de Québec, au sujet des représailles patronales et gouvernementales contre l'exercice des activités syndicạles légitimes. Déjà des barricades se dressent dans les esprits et à moins qu'on ne corrige ces graves injustices, elles se dresseront un jour dans les rues.

\section{Propos sur le travail humain}

S.S. PIE XII

Sa Sainteté le Pape Pie XII a reçu en audience, 10 janvier 1958, les délégués de la Commission consultative internationale des entrepreneurs de l'industrie chimique d'une douzaine de pays. Le Saint-Père, au cours de l'audience, a prononcé l'allocution dont nous reproduisons le texte et qui traite des aspects sociaux de l'important secteur d'activité qu'exercent les dirigeants de l'industrie chimique.

Il Nous est agréable, Messieurs, de vous recevoir à l'occasion de la rencontre amicale que vous tenez à Rome ces jours-ci, et de saluer ainsi les représentants des groupes les plus importants d'industries chimiques en Europe occidentale et en Amérique du Nord. Vos réunions ont pour but d'examiner divers sujets de 
caractère social, en particulier la question des mesures adoptées dans les usines pour protéger la santé et assurer la sécurité des travailleurs, ainsi que le problème des relations professionnelles. Vous entendez ainsi préparer la discussion des thèmes figurant au programme de la Commission des Industries Chimiques au Bureau international du Travail.

Nous ne pouvons que Nous réjouir de voir aborder ces sujets par les chefs responsables d'un secteur industriel encore ieune, et qui participe étroitement aux développements de la technique moderne. Vous vous élevez d'abord avec fermeté contre le préjugé courant, qui considère le personnel des produits chimiques comme particulièrement exposé aux maladies professionnelles et aux accidents. Les épaisses traînées de fumée fuligineuse qui s'échappent des cheminées, les odeurs désagréables, l'apparence quelque peu mystérieuse des processus chimiques, suffisent à l'imagination pour édifier mille hypothèses gratuites et avancer que ce genre d'industrie exerce des effets particulièrement néfastes sur la santé de ceux qu'elle emploie.

Aussi avez-vous désiré que des enquêtes précises et objectives fixent le nombre et l'importance des accidents et des maladies encourus par vos ouvriers. Bien que cette enquête n'ait été entreprise en Italie que depuis 1955, elle démontre aisément - selon les renseignements que vous avez bien voulu Nous transmettre que la fréquence et la gravité de ces accidents et maladies sont notablement moindres dans l'industrie chimique que pour la moyenne de toutes les autres activités industrielles. Ainsi se trouve réfutée par les faits une généralisation dépourvue de fondement réel. Cette constatation rassurante ne peut cependant légitimer un relâchement de vigilance ni faire reléguer au second plan les problèmes de sécurité et de salubrité. Peut-être certains établissements conservent-ils encore une proportion d'accidents plus élevée, par insuffisance des moyens de protection, ou par défaut de modernisation des installations: il importe donc qu'ils appliquent sans retard les mesures opportunes pour y remédier.

Mais sans aucun doute, devez-vous tendre pour l'ensemble de votre industrie à l'amélioration des conditions de travail, à la sécurité la plus grande possible de la main-d'oeuvre, à sa protection plus efficace contre les dangers inhérents au travail lui-même. ou contre ceux que provoque le manque de compétence ou la négligence. C'est là un devoir commun à tous les dirigeants d'industrie, mais Nous espérons que vous aurez à coeur de conserver et même d'accroître la marge d'écart, oui maintient vos statistiques des accidents et maladies professionnelles en dessous de la moyenne générale.

Le problème de la sécurité ne constitue d'ailleurs qu'un aspect de celui. plus large, des conditions humaines du travail en usine. La collaboration de l'employeur et de son personnel dans l'activité de production obéit à des mobiles apparemment divergents, mais dont les études sociales récentes découvrent mieux la coïncidence profonde. Le patron veut naturellement augmenter le rendement de la maind'oeuvre par une meilleure organisation de la production, tandis que l'ouvrier asvire à une participation plus large au fruit de ses efforts. matérialisé dans le salaire. Mais les chefs d'industrie, éclairés par une connaissance plus exacte des exigences réelles du travail humain, de ses facteurs psychologiques individuels et sociaux, en viennent de plus en plus, à subordonner les éléments purement économiques de la production aux impératifs issus de la nature spirituelle de l'homme, des légitimes aspirations de son esprit et de ses dispositions affectives. Les gens compétents reconnaissent que devant un travail inadapté, qui méconnaît ou avilit sa personnalité au lieu de l'épanouir, le travailleur ralentit son effort productif et réduit ainsi considérablement les avantages obtenus depuis vingt-cinq ans par la mécanisation. Des psychologues ont essayé de classer les influences nombreuses, qui déterminent le comportement de l'ouvrier devant son travail; il semble que la plus notable soit l'intérêt actif, qui fixe l'homme à sa tâche et lui donne l'impression de mettre en oeuvre ses ressources personnelles et de les développer. L'ouvrier sent alors qu'il engage non seulement ses forces musculaires, mais aussi son âme et que ses peines sont récompensées d'abord par la fierté de l'oeuvre accomplie, 
qui le grandit lui-même. Au lieu de voir uniquement dans son travail le moyen de gagner un salaire, il y découvre le sens de sa vie, la valeur de son être personnel et social.

Si déjà au simple point de vue de la productivité, cet élément mérite la sérieuse attention des chefs d'entreprises, il s'impose bien davantage à qui s'élève jusqu'au plan de la conscience humaine et de ses responsabilités absolues. Celles-ci, le Christ les a nettement exprimées, quand il déclarait, en sa qualité de Souverain Juge, que tout ce qu'on ferait au moindre des siens, c'est à lui-même qu'on le ferait (cfr. Matth. 25, 40). Respectueux des personnes et de leurs droits inaliénables, conscient de la solidarité profonde qui le relie au plus humble de ses semblables, l'homme de coeur, le chrétien surtout, ne permet pas qu'on juge les faits économiques et les situations sociales à la lumière du déterminisme de lois aveugles ou d'une évolution historique inexorable. Il souffre profondément de voir que l'ouvrier d'aujourd'hui reste trop souvent étranger à son travail, enchaîné à un labeur qui l'enserre comme un carcan, au lieu de lui donner, si modeste soit-elle, une possibilité d'épanouissement.

Nous savons, Messieurs, que vous êtes conscients de cette difficulté et soucieux d'échanger vos expériences et vos idées, pour améliorer progressivement une situation que vous n'avez pas créée vous-mêmes. N'ayez de cesse que vos entreprises puissent assurer à tout leur personnel les moyens de s'épanouir comme travailleurs et comme hommes, dans un effort productif sans doute, mais aussi profondément éducatif, qui leur donne la conscience de leur rôle social, de leur importance, de l'efficacité de leur collaboration à l'oeuvre commune. Ainsi vous approcherez-vous davantage de l'idéal proposé par l'Evangile à ceux qui sont invèstis d'une responsabilité sociale, l'idéal de la charité qui n'est point condescendance accasionnelle, mais souci constant du bien d'autrui et de son accomplissement personnel et social dans l'ordre voulu par Dieu.

En souhaitant de tout coeur que vos travaux obtiennent tous les résultats féconds que vous en attendez, et comme gage des faveurs célestes, Nous vous accordons bien volontiers Notre Bénédiction apostolique. »

Rapport du XIe Congrès des relations industrielles de Laval

\section{La stabilité de I'emploi}

- Prospérité économique et paradoxe de l'emploi - Charles Lemelin

- Le chômage struoturel et cyclique - RenÉ Tremblay

- Le chômage saisonnier et frictionnel - J.-M. Martin

- Implications sociologiques de l'instabilité de l'emploi FERNAND DUMONT

- L'expérience canadienne: l'Etat - Pierre Harvey

- L'expérience canadienne: le secteur privé de l'entreprise Eugène Forsey, R.B. MacPherson et W.M. Berry

- Aspect normatif de la stabilité de l'emploi - Maurice Tremblay

En vente $a$ :

\section{Les Presses Universitaires Laval}

28, Ste-Famille, Québec. 